\title{
CARDIOPULMONARY BYPASS, MYOCARDIAL MANAGEMENT, AND SUPPORT TECHNIQUES
}

\section{NEUROPHYSIOLOGIC MONITORING TO ASSURE DELIVERY OF RETROGRADE CEREBRAL PERFUSION}

Brian L. Ganzel, MD ${ }^{\mathrm{a}}$

Harvey L. Edmonds, Jr, $\mathrm{PhD}^{\mathrm{b}}$

John R. Pank, MD ${ }^{b}$

L. Jane Goldsmith, $\mathrm{PhD}^{\mathrm{e}}$
Background: Patients undergoing complex aortic procedures performed with deep hypothermia and circulatory arrest have a significant risk of an adverse neurologic event when the arrest period is prolonged. Retrograde cerebral perfusion appears to improve cerebral protection, although collapsed cortical veins or functional jugular venous valves may restrict flow at the frequently recommended maximum pressure of $25 \mathrm{~mm} \mathrm{Hg}$. Therefore, the purpose of this study was to demonstrate the benefit of multimodality neurophysiologic monitoring in assuring delivery of retrograde cerebral perfusion. Methods: Electroencephalography, cerebral blood flow velocity, and regional cerebral venous oxygen saturation were used to quantify the intraoperative neurophysiologic changes accompanying retrograde cerebral perfusion. The magnitude of changes was compared with those previously observed during arrest without retrograde cerebral perfusion. Results: Thirty patients underwent complex aortic procedures necessitating circulatory arrest, 22 with retrograde cerebral perfusion. The mean retrograde perfusion pressure of $40 \mathrm{~mm} \mathrm{Hg}$ ( 30 to $49 \mathrm{~mm} \mathrm{Hg}, 95 \%$ confidence interval) and flow rate of $1.2 \mathrm{~L} / \mathrm{min}(0.9$ to $1.6 \mathrm{~L} / \mathrm{min})$ necessary to achieve documented retrograde cerebral perfusion was much higher than previously recommended. During both retrograde cerebral perfusion and rewarming, cerebral oximetric monitoring guided adjustments in perfusion parameters to limit the rate of desaturation to $0.4 \%$ per minute $(0.3 \%$ to $0.6 \%$ ). With retrograde cerebral perfusion there was a rapid (1) recovery of electroencephalographic activity during rewarming ( 21 minutes [range 16 to 26 minutes]) and (2) return of consciousness after the operation (81\% [58\% to $95 \%, 95 \%$ confidence interval] awake by 12 hours). There was no transcranial Doppler evidence of cerebral edema with retrograde cerebral perfusion. Two neurologic complications occurred in the 22 patients managed with retrograde cerebral perfusion and one in the eight patients managed with arrest only. Conclusions: Multimodality neurologic monitoring assured optimal brain cooling and bihemispheric delivery of retrograde cerebral perfusion. Necessary retrograde pressure and flow were often higher than values previously reported. Avoidance of profound cerebral venous oxygen desaturation during retrograde cerebral perfusion and rewarming was associated with rapid recovery of neurologic function. (J Thorac Cardiovasc Surg 1997;113:748-57)
From the Division of Thoracic and Cardiovascular Surgery, ${ }^{\text {a De- }}$ partments of Surgery, Anesthesiology, ${ }^{\mathrm{b}}$ and Biostatistics Center, ${ }^{\mathrm{C}}$ University of Louisville School of Medicine, Louisville, Ky.

Supported in part by the Mary and Mason Rudd Endowment of Jewish Hospital, Louisville, Ky.

Read at the Seventy-sixth Annual Meeting of The American Association for Thoracic Surgery, San Diego, Calif., April 28--May 1, 1996.
Received for publication May 6, 1996; revisions requested June 13, 1996; revisions received Nov. 25, 1996; accepted for publication Dec. 23, 1996.

Address for reprints: B. L. Ganzel, MD, Department of Surgery, University of Louisville, Louisville, KY 40292

Copyright $@ 1997$ by Mosby-Year Book, Inc.

$0022-5223 / 97 \$ 5.00+0 \quad \mathbf{1 2 / 6 / 8 0 0 3 5}$ 
D eep hypothermia with circulatory arrest is a valuable technique for use during operations to correct complex aortic abnormalities. The safety and efficacy of this technique is well founded in laboratory and clinical studies. However, the probability of an adverse neurologic event is directly related to the duration of arrest. ${ }^{1}$ Retrograde cerebral perfusion (RCP) was recently reintroduced by Ueda and associates $^{2}$ in hope of increasing the safe arrest period. Although initial clinical results appear promising, ${ }^{3-6}$ objective intraoperative neurophysiologic data on the immediate effects of RCP are not well established. Furthermore, animal studies suggest possible difficulties and dangers associated with RCP. For example, both Nojima ${ }^{7}$ and $\mathrm{Usui}^{8}$ and their associates found that superior vena caval pressures above $25 \mathrm{~mm} \mathrm{Hg}$ during retrograde flow were associated with cerebral edema in canine models. Midulla and colleagues, ${ }^{9}$ using a porcine model, observed a significant pressure gradient between the superior vena cava and sagittal sinus. They concluded that without sagittal sinus pressure monitoring, definite underperfusion would have occurred in $38 \%$ of their animals.

Potential impediments to retrograde cerebral flow have also been observed clinically. First, cortical veins collapse during a sudden loss of cerebral perfusion pressure. ${ }^{10}$ Thus a zero or negative transmural venous pressure accompanying conversion of antegrade to retrograde perfusion may result in increased cerebrovenous resistance, which must be overcome to initiate RCP within the cortical mantle. Second, Okamato and coworkers ${ }^{11}$ and Imai, Hanaoka, and Kemmotsuo ${ }^{12}$ have observed competent jugular valves in some patients. Superior vena caval pressures of more than $50 \mathrm{~mm} \mathrm{Hg}$ were often required to breach these valves. These findings raise concern about the reliability and effectiveness of low-pressure RCP. Substantiating this concern, Sakahashi and colleagues ${ }^{13}$ failed to find transcranial Doppler ultrasonic evidence of RCP in $50 \%$ of their small patient sample. In accord with the previously cited animal studies, they limited caval pressure to $40 \mathrm{~mm} \mathrm{Hg}$ and flow rate to $0.5 \mathrm{~L} / \mathrm{min}$.

Before initiation of large-scale prospective clinical efficacy trials, it thus appears essential to develop monitoring methods that document the safe delivery of RCP. Therefore this retrospective cohort outcome analysis was undertaken to demonstrate the clinical application of multimodality neurophysiologic monitoring to the technique of RCP. The electroencephalogram (EEG) was used to deter- mine the individualized optimal cranial temperature for the initiation of RCP and document the return of synaptic function during rewarming. Cerebral blood flow velocity, as measured by transcranial Doppler ultrasonography, verified the successful establishment of RCP in both cerebral hemispheres. Transcranial near-infrared spectroscopic measurement of relative cerebral venous oxygen saturation in each hemisphere served to individually adjust perfusion conditions to minimize desaturation during both RCP and later rewarming with antegrade flow.

\section{Methods}

Patients. Between August 1993 and March 1996, 30 patients underwent neurophysiologic monitoring during complex aortic procedures necessitating deep hypothermic circulatory arrest. The clinical and operative notes, as well as the anesthesia and perfusion records, of all 30 patients were reviewed. All the patients had pharmacologic neurologic protection with phenytoin $(15 \mathrm{mg} / \mathrm{kg}$ intravenously) before cardiopulmonary bypass (CPB) and methylprednisolone sodium succinate $(3 \mathrm{mg} / \mathrm{kg}$ intravenously) while cooling on CPB. Twenty-two of the 30 patients had RCP for additional cerebral protection during arrest. The neurophysiologic monitoring and clinical outcome data of these 22 patients were then related to the data of eight patients who required circulatory arrest but did not undergo RCP.

The CPB circuit and priming solution, the cooling and rewarming rates, and the anesthesia protocols were similar in the two groups. Twenty-seven operations were performed through median sternotomies with femoral artery and right atrial cannulation. The remaining three operations were performed through thoracotomies with femoral artery and femoral vein cannulation. The appropriate arrest temperature was achieved when a flat-line EEG was obtained. RCP was accomplished by perfusing oxygenated blood at the arrest temperature via a $22 \mathrm{~F}$ wire-reinforced venous return cannula placed into the superior vena cava, which was snared with a tourniquet (Fig. 1). Bilateral arm tourniquets were inflated to $60 \mathrm{~mm} \mathrm{Hg}$ during RCP. The central venous pressure was measured in the superior vena cava.

EEG. Cerebrocortical function was objectively characterized by means of quantitative EEG. Cerebral biopotentials were recorded with gold cup electrodes placed bilaterally on the patient's forehead and mastoid. Fourier spectral analysis of the EEG (A-1000, Aspect Medical Systems, Natick, Mass., Spectrum 32, Cadwell Labs, Kennewick, Wash., or NeuroTrac II, Moberg Medical, Ambler, $\mathrm{Pa}$.) produced simple numeric indices suitable for characterizing changes in the complex waveforms. Amplitude of the signal was determined by total power in picowatts in successive 2-second epochs. A flat-line pattern was defined as an amplitude of less than 10 picowatts (i.e., signal-to-noise ratio of $<10: 1$ ) in more than $35 \%$ of epochs per minute. 


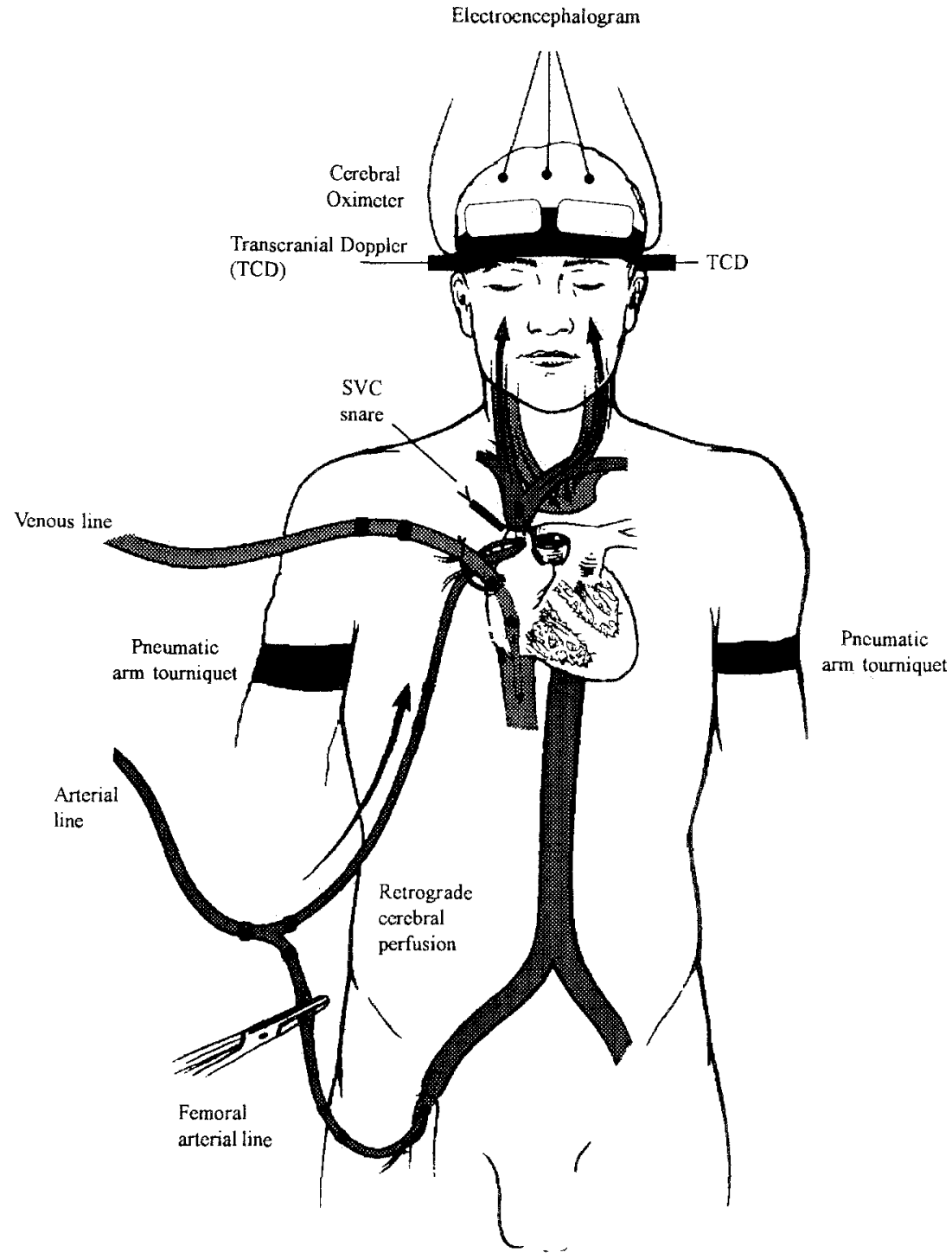

Fig. 1. Technique of RCP and neurophysiologic monitoring during deep hypothermia and circulatory arrest. SVC, Superior vena cava. (Courtesy of Somanetics Corporation, Troy, Michigan.)

Measurement of cerebral blood flow velocity. Actual retrograde flow through the middle cerebral arteries was verified continuously and noninvasively by transcranial Doppler ultrasonography. Two $2 \mathrm{mHz}$ pulse-wave ultrasonography transducers were positioned with a commercially available fixation device (Medasonics, Fremont, Calif.) over the left and right temporal regions. These transducers insonated the portion of the middle cerebral arteries near their juncture with the ipsilateral anterior cerebral artery. A color spectral analyzer (NeuroGuard, Medasonics) displayed the flow velocity profile on a VGA high-resolution color graphics monitor (Mitsuba Electronics, Tokyo, Japan). The technical specifications of the monitor's capabilities of measuring low flow velocity are described in Appendix 2. Through a VGA-NTSC video hyperconverter (PC Video Conversion, San Jose, Calif.), transcranial Doppler recordings from the entire operation were videotaped for subsequent review. Key spectral segments illustrating changes in cerebral blood flow velocity were also stored digitally and printed as black and white images. Cerebral blood flow velocity was quantified from the upper edge of the velocity spectrum. A reversal of flow direction was signified by spectral inversion.

Measurement of regional cerebral venous oxygen saturation. The adequacy of the retrograde flow rate was assessed according to relative changes in cerebrovenous oxygen saturation with transcranial near-infrared spectroscopy. Self-adhesive patches, which contained an infrared light-emitting diode and two distant sensors $(30 \mathrm{~mm}$ [scalp] and $40 \mathrm{~mm}$ [scalp plus brain]) were fixed on the left and right sides of the patient's forehead. Cerebrovenous oxygen saturation was calculated from the differential 
signal obtained from these two sensors, expressed as the venous-weighted percent oxygenated hemoglobin. The cerebrovenous oxygen saturation trend was displayed on an infrared spectrophotometer (INVOS 3100A, Somanetics, Troy, Mich.). This trend represented a temperature-corrected average of 16.5 seconds of data updated every 3.3 seconds. Data points comprising the moving average trend were stored continuously on floppy disk every 10 seconds.

Anesthesia and monitoring protocol. Anesthesia was provided by a single anesthesiologist (J.R.P.) using a standardized protocol based on moderate-dose opioid infusion with continuous isoflurane supplementation. All monitoring was performed by one clinical neurophysiologist (H.L.E.). Neurologic monitoring had eight objectives, which were applied consistently. The first was to ensure adequate bihemispheric perfusion during surgical exposure, vessel cannulation, and the initiation of CPB. The second was the appearance of a flat-line EEG, which individualized the nasopharyngeal temperature for arrest. Third, transcranial Doppler ultrasonography facilitated bilateral RCP by prompting adjustments in perfusion cannula position, snare tension, and caval pressure (up to $60 \mathrm{~mm} \mathrm{Hg}$ ) to produce graphic and acoustic evidence of reversed flow. Fourth, optimal RCP flow was guided by cerebral oximetry. Flow was increased to a maximum of $2.8 \mathrm{~L} / \mathrm{min}$ to minimize cerebrovenous oxygen desaturation. With high flow, continued rapid desaturation prompted a decrease in perfusate temperature. Fifth, transcranial Doppler ultrasonography verified return of antegrade perfusion through both middle cerebral arteries. Sixth, the EEG provided an objective indicator of the return of cerebrocortical function. Seventh, cerebrovenous oxygen desaturation during rewarming was managed with a pharmacologically increased cerebral perfusion pressure and cerebral metabolic suppression with a propofol infusion titrated to return saturation to the prebypass baseline. Eighth, neurologic monitoring facilitated the application of standardized anesthetic delivery by avoiding episodes of inadequate or excessive anesthesia.

Postoperative care. Postoperative sedation for all patients was standardized under the direction of a single surgeon (B.L.G.). Neurologic outcomes were determined by documented neurologic events and by the postoperative level of consciousness scoring, which was obtained from the intensive care unit nursing records. The percent of each group who were awake and alert 12 hours after the operation was calculated from these records. The percentages of patients who were extubated within 18 hours, discharged from the intensive care unit within 24 hours, and discharged from the hospital within 10 days were also determined for patients with and without RCP.

Statistical analysis. Normally distributed data were described by the mean and $95 \%$ confidence interval. Ordinal (scalar) values and data that were not normally distributed were expressed as proportions (i.e., percent affected, with $95 \%$ confidence intervals). The confidence intervals for binomial proportions were calculated with personal computer-based StatXact software (Cytel Software, Cambridge, Mass.). Unless otherwise indicated, all numbers in parentheses signify $95 \%$ confidence intervals.
Table I. The diagnoses of patients with RCP versus patients with arrest only

\begin{tabular}{lll}
\hline \multicolumn{1}{c}{ Diagnosis } & RCP & Arrest \\
\hline Primary & & \\
Annuloaortic ectasia & 8 & 3 \\
Ascending aneurysm & 3 & 2 \\
Arch aneurysm & 2 & 1 \\
Descending aneurysm & 0 & 1 \\
Thoracoabdominal aneurysm & 0 & 1 \\
Proximal aortic dissection & & \\
$\quad$ Acute & 5 & 0 \\
$\quad$ Chronic & 4 & 0 \\
Secondary* & & \\
Coronary artery disease & 5 & 4 \\
Arch aneurysm & 3 & 3 \\
Descending aneurysm & 0 & 1 \\
Innominate aneurysm & 1 & 1 \\
Carotid aneurysm & 1 & 0 \\
Aortic stenosis & 1 & 0 \\
Mitral regurgitation & 1 & 1 \\
\hline
\end{tabular}

Antest, Deep hypothermia and circulatory arrest; $R C P$, retrograde cerebral perfusion.

"Some patients had multiple secondary diagnoses.

\section{Results}

Patient characteristics. The clinical characteristics of both groups are summarized in Tables I and II. The RCP group comprised 22 patients (male/ female ratio 16:6) with a mean age of 61 years (range 23 to 81 years). The arrest-only group consisted of eight patients (male/female ratio 5:3) with a mean age of 62 years (range 36 to 76 years). The incidence of previous sternotomy, diabetes mellitus, hypertension, and preoperative renal insufficiency was comparable in the two groups. Appendix 1 describes the temporal sequence of the use of RCP, which began with the sixth case of the series. Beginning in July 1994, the retrograde technique was attempted in all patients except those requiring a left thoracotomy. Despite a preponderance of arrest-only treatment in the early phase of the study, the demographics and clinical management of all patients were similar. The lack of difference and low variability in arrest times between arrest-only and RCP groups testifies to the uniformity of surgical technique during the study.

Neurophysiologic monitoring data. The middle cerebral arteries of one female patient could not be insonated because of the absence of a temporal ultrasonic window caused by hyperostosis. A loss of transcranial Doppler signal at aortic crossclamping in two patients with acute aortic dissections identified a malperfusion syndrome, necessitating a change in cannulation site. Nasopharyngeal temper- 
Table II. The operations of patients with RCP versus patients with arrest only

\begin{tabular}{lrc}
\multicolumn{1}{c}{ Type of operation } & $R C P$ & Arrest \\
\hline Primary & 13 & \\
Aortic valved conduit* & 2 & 1 \\
Repair ascending aneurysm* & 2 & 1 \\
Repair arch aneurysm & 0 & 1 \\
Repair descending aneurysm $\dagger$ & 0 & 1 \\
Repair thoracoabdominal aneurysm $\dagger$ & & \\
Repair aortic dissection & 4 & 0 \\
$\quad$ Acute* & 1 & 0 \\
$\quad$ Chronic* & & \\
Secondary & 5 & 3 \\
Repair arch aneurysm & 1 & 1 \\
Repair innominate aneurysm & 1 & 0 \\
Repair carotid aneurysm & 0 & 1 \\
Repair descending aneurysm & 4 & 4 \\
Coronary artery bypass & 1 & 0 \\
Aortic valve replacement & 1 & 1 \\
Mitral valve replacement & \\
\hline
\end{tabular}

Arrest, Deep hypothermia and circulatory arrest; $R C P$, retrograde cerebral perfusion.

*Open distal anastomosis.

†Open proximal anastomosis.

$\doteqdot$ Some patients had multiple secondary operations.

ature required to maintain a flat-line EEG varied widely from $8^{\circ}$ to $22^{\circ} \mathrm{C}$.

The duration of circulatory arrest was similar in both the RCP and arrest-only groups (Table III). Furthermore, the nasopharyngeal temperature at the onset of circulatory arrest in the two groups was similar (Table III). Despite these similarities, there were group differences in physiologic responses.

Appendix 1 provides for each patient the antegrade cerebral blood flow velocity before arrest and the initial retrograde velocity. In patients treated before June 1995, we were unable to reliably detect RCP with transcranial Doppler ultrasonography. The $150 \mathrm{~Hz}$ high-pass filter used in the original Doppler units prevented detection of flow velocities of less than $6 \mathrm{~cm} / \mathrm{sec}$. Subsequently, a $75 \mathrm{~Hz}$ filter was introduced that permitted detection of velocities as low as $3 \mathrm{~cm} / \mathrm{sec}$. With this improved Doppler system (see Appendix 2), retrograde signals were detected in 16 of 18 cases. The group mean initial retrograde velocity was $7 \mathrm{~cm} / \mathrm{sec}(5$ to $9 \mathrm{~cm} / \mathrm{sec}$ ) compared with the prearrest antegrade value of 22 $\mathrm{cm} / \mathrm{sec}(19$ to $25 \mathrm{~cm} / \mathrm{sec})$. A consistent close relationship between retrograde velocity/signal intensity and pump flow verified that the small ultrasonic signals actually reflected reverse flow through the middle cerebral arteries and were not due to a low-frequency artifact.
Table III. Intraoperative neurophysiologic monitoring results

\begin{tabular}{lcc}
\hline & $\begin{array}{c}R C P \\
(n=22)\end{array}$ & $\begin{array}{c}\text { Arrest } \\
(n=8)\end{array}$ \\
\hline $\begin{array}{l}\text { Circulatory arrest } \\
\text { Duration (min) } \\
\text { Temperature }\left({ }^{\circ} \mathrm{C}\right)\end{array}$ & $26(22-30)^{*}$ & $24(15-33)$ \\
$\begin{array}{l}\text { Regional cerebral venous oxygen } \\
\text { saturation decrease }\end{array}$ & $17(16-19)$ & $17(14-20)$ \\
$\quad \begin{array}{l}\text { Percent } \\
\text { Rate (percent/min) }\end{array}$ & $11(7-14)$ & $21(9-33)$ \\
Electroencephalography & $0.4(0.3-0.6)$ & $0.9(0.4-1.5)$ \\
$\quad$ Return (min) & $21(16-26)$ & $55(21-90)$ \\
\hline $\begin{array}{l}\text { Arrest, Deep hypothermia and circulatory arrest; } R C P, \text { retrograde cerebral } \\
\text { perfusion. } \\
\text { * Mean (95\% confidence interval of the mean). }\end{array}$
\end{tabular}

The average superior vena caval pressure of 40 $\mathrm{mm} \mathrm{Hg} \mathrm{(30} \mathrm{to} 49 \mathrm{~mm} \mathrm{Hg}$ ), necessary to establish Doppler-verified RCP, was much higher than the previously recommended maximum of $25 \mathrm{~mm} \mathrm{Hg}$. Additionally, the retrograde flow rate needed to prevent initial rapid $(>1 \% / \mathrm{min})$ cerebral oxygen desaturation was $1.2 \mathrm{~L} / \mathrm{min}(0.9$ to $1.6 \mathrm{~L} / \mathrm{min})$. PostCPB end-diastolic flow velocity of $29 \mathrm{~cm} / \mathrm{sec}$ ( 23 to 35 $\mathrm{cm} / \mathrm{sec}$ ) in the RCP group was similar to the pre-CPB baseline value of $22 \mathrm{~cm} / \mathrm{sec}(16$ to $28 \mathrm{~cm} / \mathrm{sec})$.

The extent of cerebral oxygen desaturation (i.e., percent decrease from prearrest baseline) appeared somewhat larger in arrest-only patients (Table III). Similarly, the average rate of desaturation in the arrest-only group was nearly double that seen in the RCP group (Table III).

Clearly, the cerebral oximetric measurements reflected dynamic metabolic processes during RCP and were not the result of stagnant cerebral venous pooling. The cerebrovenous oxygen saturation values consistently responded appropriately to measures designed to further reduce the rate of cerebrovenous oxygen desaturation during RCP. After the transcranial Doppler-confirmed establishment of bilateral RCP, occasional continued rapid desaturation was abated by an increase in pump flow or decrease in perfusate temperature. Patients with $\mathrm{RCP}$ also had a more rapid return of cerebrocortical function. Continuous EEG activity returned more quickly with RCP (Table III).

Postoperative outcome results. As expected, the influence of RCP on recovery was most apparent in its earliest phases (Table IV). Patients managed with $\mathrm{RCP}$ regained consciousness more quickly than those with arrest only. No patient in the arrest-only 
Table IV. Postoperative outcome results

\begin{tabular}{llccc}
\hline & \multicolumn{2}{c}{ RCP } & & \multicolumn{2}{c}{ Arrest } \\
\cline { 2 - 5 } Variable & $n$ & $\%$ & & $n$ \\
\hline Awake by $12 \mathrm{hr}$ & 22 & $81(58-95)^{*}$ & 8 & $0(0-31)$ \\
Extubated by $18 \mathrm{hr}$ & 19 & $42(20-67)$ & 8 & $13(0-53)$ \\
ICU discharge within 24 hr & 19 & $32(13-57)$ & 8 & $0(0-21)$ \\
Hospital discharge within 10 days & 19 & $47(24-71)$ & 8 & $37(19-76)$ \\
\hline
\end{tabular}

Amest, Deep hypothermia and circulatory arrest; $I C U$, intensive care unit; $R C P$, retrograde cerebral perfusion.

"Ninety-five percent confidence interval of the proportion.

group was awake and alert within the first 12 postoperative hours, compared with $81 \%$ in the RCP group. Although $42 \%$ of RCP-managed patients were extubated within 18 hours versus $13 \%$ of those without RCP, their respective $95 \%$ confidence limits overlapped (Table IV). Similar differences were seen in the fraction of patients discharged from the intensive care unit within 24 hours (32\% with RCP vs $0 \%$ without RCP) and from the hospital within 10 days ( $47 \%$ with RCP vs $37 \%$ without RCP) (Table IV). There were two neurologic complications in the RCP-managed patients compared with one in the arrest-only group.

Two deaths occurred in the RCP group, one of which was due to a neurologic event. This patient had an acute type I aortic dissection and a history of a preoperative stroke. She died 8 days after the operation of complications from an intraoperative stroke. The second patient died 81 days after the operation of complications of acquired immunodeficiency syndrome, which was diagnosed in the postoperative period after the repair of an acute type I aortic dissection. Data from both patients were included in the level of consciousness scoring and neurologic event analysis; however, neither patient's data were included in the analysis of extubation time, intensive care unit stay, or hospital length of stay.

\section{Discussion}

The applicability of comparative inferential statistics in retrospective cohort studies is often diminished by imbalance in patient assignment and an absence of true randomization. This report is no exception. As an account of our initial experience with RCP guided by neurologic monitoring, it is not a truly randomized clinical trial. So that we could gain experience with the retrograde technique quickly, patients were not randomized to RCP or arrest only and the sample sizes were both small and unequal. Inasmuch as these inadequacies may result in statistical bias, caution is urged in the comparison of group data from the RCP and arrest-only groups. In particular, we note that the majority of the arrest-only patients were studied first.

Study design limitations notwithstanding, these results suggest that, with the guidance of multimodality neurologic monitoring, RCP can be reliably and safely delivered. Because transcranial Doppler end-diastolic flow velocity is a sensitive indicator of developing intracranial hypertension, ${ }^{14,15}$ it provided objective evidence that cerebral edema was absent in patients treated with retrograde pressures and flows higher than those previously recommended. Postbypass end-diastolic flow velocities were similar to their respective prebypass baselines.

Regional cerebral venous oxygen saturation, as measured noninvasively by near-infrared spectroscopy, detected changes in oxygen saturation from within the adult cerebral cortex. ${ }^{16}$ Desaturation changes detected by this technique indicate a failure of increased cerebral oxygen extraction to keep pace with falling delivery or increased metabolic demand. ${ }^{17}$ We are unaware of any reports that document regional cerebral venous oxygen desaturation from other physiologic causes. Absolute saturation values are influenced by many variables, and normative values have not been established. Clinical applications of cerebral oximetry, therefore, have relied on relative changes expressed as percent deviation from baseline. Our study demonstrated a significantly lower relative total desaturation and a lower rate of desaturation in patients receiving $\mathrm{RCP}$. This indicates that RCP provided at least part of the oxygenation necessary for cerebral metabolic demands at these temperatures. Our findings are thus in agreement with those of Deeb and associates, ${ }^{5}$ who proposed that cerebral oximetry-monitored RCP appears to extend the safe arrest time. Transcranial Doppler ultrasonography is necessary to document RCP flow, whereas the oximetry data indicate the effectiveness of a given flow in main- 
taining oxygenation. This combined monitoring can detect cerebral ischemia if desaturation occurs during RCP, despite flow documented by transcranial Doppler ultrasonography. Therapeutic options include further cooling of the retrograde perfusate, increasing RCP flows, increasing carbon dioxide tension, and pharmacologic interventions to suppress cerebral metabolic activity.

Cerebral oximetry also provides important information during systemic rewarming after circulatory arrest. During resumption of antegrade cerebral flow and rewarming, the normal linear relationship between cerebral blood flow and brain temperature may be disrupted (flow-metabolism uncoupling). Oxygen desaturation that occurs during rewarming as a result of uncoupling is particularly dangerous, because the brain is now metabolically hyperactive and at increased risk for ischemic insult. This condition may require metabolic suppression and neuroprotectant intervention to avoid a poor neurologic outcome.

The optimal cranial temperature for arrest has not been established. Coselli and coworkers ${ }^{18}$ proposed that adequate systemic cooling occurs when a flat-line EEG is obtained and provides optimal brain protection for arrest. Others ${ }^{19}$ have proposed systemic cooling to specific lower temperatures, because there may be a poor correlation between brain temperature and EEG activity. In our study, the temperature at which the EEG waveform became flat varied greatly among patients $\left(8^{\circ} \mathrm{C}\right.$ to $\left.22^{\circ} \mathrm{C}\right)$ and confirms the findings reported by Coselli's group. ${ }^{18}$ Cerebral oximetry data during cooling provided further information on the appropriate arrest temperature. We found that as cooling progressed, cerebral oxygen saturation increased because cerebral metabolic activity decreased more rapidly than luxuriant cerebral blood flow. The oxygen saturation trend reached a plateau near the temperature associated with a flat-line EEG. Because the benefit of hypothermia is generally attributed to synaptic depression, further cooling beyond a flat-line EEG or oxygen saturation plateau, therefore, would not be expected to increase cerebral protection. Cooling to the same determined fixed temperature may not be appropriate for all patients and could lead to excessive cooling, which has its own potential problems including severe coagulopathies. Instead, neurophysiologic monitoring data can be used to objectively determine the optimal arrest temperature and can also define efficacious RCP parameters of brain temperature, arterial carbon dioxide tension, pump flow rate, and superior vena caval pressures for partial cerebral protection during arrest.

Our results suggest that when compared with arrest only, adequate RCP provides patients with a more rapid return of continuous EEG activity and an earlier return of consciousness in the postoperative period. The value of quantitative EEG in objectively assessing cerebral injury after arrest has been demonstrated. ${ }^{20,21}$ However, the retrospective and subjective nature of our return-to-conscious data requires a more cautious interpretation. Caution is especially warranted inasmuch as the determinations were made by several members of the nursing staff, rather than by a single rater. Validation of these latter findings will require prospective blinded assessment.

The low overall incidence of documented adverse neurologic events in both groups limits its usefulness as an outcome measure of RCP benefit. This is particularly true because the mean circulatory arrest times were relatively short in both groups and well within the arrest time considered to be safe. However, our findings are consistent with the recent observations of Deeb and associates, ${ }^{5}$ who noted a less than $10 \%$ neurologic deficit incidence with a mean RCP time of 63 minutes (range 35 to 128 minutes). These excellent results were in stark contrast with their earlier dismal experience with arrestonly periods exceeding 60 minutes.

Not surprisingly, the other outcome measures reflecting extubation time, intensive care unit length of stay, and hospital length of stay did not indicate improved neurologic protection. These outcome measures are relatively insensitive, however, and dependent on a multitude of postoperative factors unrelated to the use of RCP.

In summary, under the guidance of multimodality neurologic monitoring, $\mathrm{RCP}$ using relatively high pressure and flow appears to be at least as safe as circulatory arrest alone. There was no neurophysiologic or clinical evidence of either cerebral edema or hemorrhage with RCP. The diminished cerebral oxygen desaturation in RCP-managed patients was associated with a more rapid return of cerebrocortical function both during and after the operation. Thus the theoretical concerns and technical difficulties associated with RCP delivery have been largely resolved. It now appears appropriate to formulate a multicenter prospective study of RCP efficacy using multimodality neurologic monitoring to standardize and optimize the technique of retrograde perfusion. 


\section{REFERENCES}

1. Ergin MA, Galla JD, Landsman SL, et al. Hypothermic circulatory arrest in operations on the thoracic aorta: determinants of operative mortality and neurologic outcome. J Thorac Cardiovasc Surg 1994;107:788-99.

2. Ueda Y, Miki S, Kusuhara K, et al. Surgical treatment of aneurysm or dissection involving the ascending aorta and aortic arch, utilizing circulatory arrest and retrograde perfusion. J Cardiovasc Surg 1990;31:553-58.

3. Bavaria JE, Woo YJ, Hall RA, Carpentier JP, Gardner TJ. Retrograde cerebral and distal aortic perfusion during ascending and thoracoabdominal aortic operations. Ann Thorac Surg 1995;60:345-52.

4. Lytle BW, McCarthy PM, Meaney KM, Stewart RW, Cosgrove DM. Systemic hypothermia and circulatory arrest combined with arterial perfusion of the superior vena cava: effective intraoperative cerebral protection. J Thorac Cardiovasc Surg 1995;109:738-43.

5. Deeb GM, Jenkins E, Bolling SF, et al. Retrograde cerebral perfusion during bypothermic circulatory arrest reduces neurologic morbidity. J Thorac Cardiovasc Surg 1995;109:259-68.

6. Takamoto S, Matsuda T, Horada M, et al. Simple hypothermic retrograde cerebral perfusion during aortic arch surgery. J Cardiovasc Surg 1992;33:560-7.

7. Nojima T, Magara T, Nakajima Y, et al. Optimal perfusion pressure for experimental retrograde cerebral perfusion. J Card Surg 1994;9:548-59.

8. Usui A, Oohara K, Liu T-L, et al. Determination of optimum retrograde cerebral perfusion conditions. J Thorac Cardiovasc Surg 1994;107:300-8.

9. Midulla PS, Gandsas A, Sadeghi AM, et al. Comparison of retrograde cerebral perfusion to antegrade cerebral perfusion and hypothermic circulatory arrest in a chronic porcine model. J Card Surg 1994;9:560-75.

10. Lasjaunias $P$, Berenstein A. Surgical neuroangiography. Berlin: Springer-Verlag, 1990:304-5.

11. Okamato H, Sato K, Matsuura A, et al. Selective jugular cannulation for safer retrograde cerebral perfusion. Ann Thorac Surg 1993;55:538-40.

12. Imai $M$, Hanaoka $Y$, Kemmotsuo. Valve injury: a new complication of internal jugular vein cannulation. Anesth Analg 1994;78:1041-6.

13. Sakahashi H, Hashimoto A, Aomi S, et al. Transcranial Doppler measurement of middle cerebral artery blood flow during continuous retrograde cerebral perfusion. J Jpn Assoc Thorac Surg 1994;42:1851-7 (Japanese with English summary).

14. Vander Linden J, Wesselen O, Ekroth R, Tyden H, Von Ahn H. Transcranial Doppler-estimated versus thermodilutionestimated cerebral blood flow during cardiac operations. J Thorac Cardiovase Surg 1991;102:95-102.

15. Newell DN, Aaslid R, Stooss R, Revlen HJ. The relationship of blood velocity fluctuations to intracranial pressure $\mathrm{B}$ waves. J Neurosurg 1992;76:415-21.

16. Hongo $\mathrm{K}$, Kobayashi S, Okudera $\mathrm{H}$, et al. Noninvasive cerebral optical spectroscopy: depth-resolved measurements of cerebral hemodynamics using indocyanine green. Neurol Res 1995;17:89-93.

17. Ausman II, McCormick PW, Stewart M, et al. Cerebral oxygen metabolism during hypothermic circulatory arrest in humans. J Neurosurg 1993;79:810-5.

18. Coselli JS, Crawford ES, Beall AC Jr, et al. Determination of brain temperatures for safe circulatory arrest during cardiovascular operations. Ann Thorac Surg 1988;45:638-42.

19. Ergin MA, Griepp EB, Lansman SL, et al. Hypothermic circulatory arrest and other methods of cerebral protection during operations on the thoracic aorta. J Card Surg 1994;9: 525-37.

20. Mezrow CK, Midulla PS, Sadeghi A, et al. Quantitative electroencephalography: a method to assess cerebral injury following hypothermic circulatory arrest. J Thorac Cardiovasc Surg 1995;109:925-34.

21. Mezrow CK, Midulla PS, Sadeghi A, et al. Evaluation of cerebral metabolism and quantitative electroencephalography following circulatory arrest and low-flow cardiopulmonary bypass at different temperatures. J Thorac Cardiovasc Surg 1994;107:1006-19.

\section{Discussion}

Dr. Randall B. Griepp (New York, N.Y.). I agree that the early appearance of EEG activity after circulatory arrest is a reliable and objective test of the adequacy of cerebral protection and correlates in animal studies well with neuropathology. I further agree that the early reappearance of EEG activity in your patient who had RCP is encouraging. Your work further documents the efficacy of $\mathrm{RCP}$ in reducing cerebral ischemia during interruption of antegrade perfusion, and, to my knowledge, it is the first routine documentation of retrograde flow in the middle cerebral artery in human beings during RCP. It also further documents the utility of near-infrared spectroscopy in monitoring the effect of RCP.

I have several questions. The mean value of the central venous pressure when retrograde flow signals were apparent in the middle cerebral artery was $39 \mathrm{~mm} \mathrm{Hg}$, a level that is quite high. What was the maximum value? Perhaps it is much safer to go higher than we have previously thought. You mentioned that the end-diastolic flow in the middle cerebral artery after CPB did not change in the RCP-treated patients from the preoperative value. I am curious as to whether it did in the patients treated with hypothermic circulatory arrest alone.

The issue of when to discontinue CPB and begin the period of circulatory arrest was mentioned as the point at which brain cerebral oxygen saturation was maximum and EEG silence occurred. Many of us who follow jugular bulb venous saturation use this as an indicator of when the brain is saturated. Do you have correlative data between the near-infrared spectroscopy and the jugular bulb saturation?

I have one further question. Most of the monitoring modalities that you have advocated involve the anterior cortex or perhaps the parietal cortex. Do you have any suggestions as to how to monitor adequately the posterior cerebral circulation? As you know, this may receive retrograde flow through pathways other than those that supply the anterior brain.

Dr. Ganzel. The maximum central venous pressure rate or the pressure that we saw was $49 \mathrm{~mm} \mathrm{Hg}$. Quite honestly, earlier in our experience we probably would not have had the courage to go to that level, but as we gained more confidence in the technique over time, we went to higher pressures.

Regarding the end-diastolic flow in the patients who 
were treated with circulatory arrest, we did not analyze those results. I agree that EEG silence is not necessarily the only way to determine the level of cooling before circulatory arrest, and while not analyzed, we saw a trend in plateauing of the cerebral oximetry data that seemed to correlate with EEG silence. We would see EEG silence at the same time that the cerebral oximeter would plateau at a certain value. I agree that we are monitoring the anterior circulation, and certainly the posterior is vulnerable. Unfortunately, we haven't any tricks in that area.

Dr. William A. Baumgartner (Baltimore, Md.). The addition of transcranial Doppler ultrasonography clearly provides the monitoring adjunct in assessing the adequacy of the methods used for neural protection.

I have three questions. First, in addition to the incidence of stroke, do you have information on the incidence of neurocognitive deficits observed in your patient groups and whether there is a difference between those groups? Second, do you have an opinion as to the temperature degree to maintain patients in the postoperative state, inasmuch as there is some laboratory evidence suggesting that maintaining patients in a cooler state before the operation leads to fewer neural problems? Third, based on our laboratory model and our past experience with clinical procurement of the heart-lung block by deep hypothermia and circulatory arrest, when the temperature was decreased to less than $10^{\circ} \mathrm{C}$ we saw a fair amount of pulmonary injury. I noticed in your abstract that the lowest temperature was around $8^{\circ} \mathrm{C}$. Did you see any pulmonary complications and did you see a correlation with the degree of temperature lowering?

Dr. Ganzel. We did not assess the more sensitive neurocognitive testing in our patients, although clearly that is something we would like to have done. The pulmonary dysfunction that you described was not really investigated, although we would like to see the patients normothermic when they leave the operating room. We have not made an attempt to keep them cool in the postoperative period. Some cooling occurs from the time they are weaned from bypass, but we have not made a conscious effort to keep them cool.

Dr. John E. Connolly (Irvine, Calif.). This is a very interesting technique to evaluate protection during arrest under profound hypothermia. However, I think the most accurate way to evaluate the time of safe cerebral ischemia under profound hypothermia is to know exactly what the brain temperature is. We know that in carotid surgery the EEG is helpful, but the cerebral circulation can be evaluated more accurately in the awake patient. Some years ago we did extensive work on dogs under profound hypothermia and circulatory arrest in a paper that we called "Bloodless Surgery," and we postulated at that time that all types of surgery, including operations for thoracoabdominal aneurysms, might ultimately be best performed under profound hypothermia and circulatory arrest if we knew what the brain temperature was at the time of arrest. In these animals, the brain temperature was monitored directly with a fine thermistor needle inserted in the brain. This technique subsequently has been widely used by neurosurgeons for cerebrovascular lesions, and it has been found that a thin needle thermistor probe does not damage the brain. We believe that the only absolutely reliable way to evaluate the length of time that you can operate with circulatory arrest under profound hypothermia is to make a small trephine in the skull through which a small thermistor needle is inserted into a frontal lobe. We found that in dogs, and I think you can extrapolate pretty well from dogs, because they do not tolerate ischemia as well as human beings, that if the brain temperature is $20^{\circ} \mathrm{C}$, an hour of arrest is safe; if the brain temperature is $15^{\circ} \mathrm{C}, 2$ hours of arrest is safe. I would like to ask the authors what they think about this approach to monitoring brain temperature.

Dr. Ganzel. It would certainly be ideal to know the actual temperature of the brain. Given the present climate of the practice of medicine, it would be somewhat difficult for us to advocate performing that technique in human beings, but it is an intriguing idea.

Appendix 1. Intraoperative transcranial Doppler monitoring results

\begin{tabular}{|c|c|c|c|c|c|}
\hline & & 7igh-pa. & Antegrade & Retrograde & \\
\hline Date & $\begin{array}{c}R C P \\
\text { attempt }\end{array}$ & $\begin{array}{l}\text { filter } \\
(H z)\end{array}$ & $\begin{array}{l}\text { velocity } \\
(\mathrm{cm} / \mathrm{sec})\end{array}$ & $\begin{array}{l}\text { velocity } \\
(\mathrm{cm} / \mathrm{sec})\end{array}$ & Comment \\
\hline $8 / 9 / 93$ & No & 150 & 15 & 0 & \\
\hline 9/9/93 & No & 150 & 23 & 0 & \\
\hline $9 / 13 / 93$ & No & 150 & 15 & 0 & \\
\hline $10 / 8 / 93$ & No & 150 & 10 & 0 & \\
\hline $12 / 17 / 93$ & No & 150 & 20 & 0 & \\
\hline $2 / 28 / 94$ & Yes & 150 & 15 & 0 & \\
\hline $3 / 21 / 94$ & No & 150 & 12 & 0 & $\begin{array}{l}\text { Left } \\
\text { thoracotomy }\end{array}$ \\
\hline $7 / 13 / 94$ & Yes & 150 & 23 & 0 & \\
\hline $7 / 21 / 94$ & Yes & 150 & 20 & 0 & \\
\hline $6 / 16 / 95$ & Yes & 75 & 19 & 8 & \\
\hline $9 / 6 / 95$ & Yes & 75 & 30 & 7 & \\
\hline $9 / 13 / 95$ & Yes & 75 & 15 & 5 & \\
\hline $9 / 20 / 95$ & Yes & 75 & 38 & 0 & \\
\hline $9 / 28 / 95$ & Yes & 75 & 20 & 9 & \\
\hline $9 / 29 / 95$ & No & 75 & 11 & 0 & $\begin{array}{l}\text { Left } \\
\text { thoracotomy }\end{array}$ \\
\hline $10 / 31 / 95$ & Yes & 75 & 33 & 9 & \\
\hline $11 / 18 / 95$ & Yes & 75 & 20 & 5 & \\
\hline $11 / 21 / 95$ & Yes & 75 & 29 & 8 & \\
\hline $12 / 11 / 95$ & Yes & 75 & 18 & 9 & \\
\hline $12 / 18 / 95$ & Yes & 75 & 20 & 8 & \\
\hline $12 / 19 / 95$ & Yes & 75 & 16 & 11 & \\
\hline $1 / 5 / 96$ & Yes & 75 & 10 & 8 & \\
\hline $1 / 10 / 96$ & No & 75 & 20 & 0 & $\begin{array}{l}\text { Left } \\
\text { thoracotomy }\end{array}$ \\
\hline $1 / 13 / 96$ & Yes & 75 & 27 & 9 & \\
\hline $1 / 19 / 96$ & Yes & 75 & 15 & 4 & \\
\hline $1 / 23 / 96$ & Yes & 75 & 30 & 7 & \\
\hline $1 / 25 / 96$ & Yes & 75 & NA & NA & $\begin{array}{l}\text { No ultrasonic } \\
\text { window }\end{array}$ \\
\hline $1 / 26 / 96$ & Yes & 75 & 14 & & \\
\hline $1 / 31 / 96$ & Yes & 75 & 20 & 16 & \\
\hline $3 / 11 / 96$ & Yes & 75 & 23 & 10 & \\
\hline
\end{tabular}

$R C P$, Retrograde cerebral perfusion; antegrade, prearrest antegrade blood flow velocity; $N A$, not applicable. 


\section{Appendix 2}

Ultrasonic measurement of retrograde flow velocity. Ultrasonic velocity resolution is determined by the pulse repetition frequency (PRF) and the number of points in the fast Fourier transformation (FFT) of the instantaneous red blood cell velocity spectrum within the insonation sample volume. At the lowest PRF of $3900 \mathrm{~Hz}$ the maximum detectable Doppler shift frequency is PRF/2 or $1950 \mathrm{~Hz}$. From the equation

$$
\mathrm{v}=\frac{\mathrm{F}_{\mathrm{d}} \mathrm{C}}{2 \mathrm{~F}_{0}}
$$

where $\mathrm{v}=$ Doppler velocity, $\mathrm{F}_{\mathrm{d}}=$ Doppler frequency, $\mathrm{C}=$ brain acoustic velocity $(154 / 000 \mathrm{~cm} / \mathrm{sec})$, and $F_{o}=$ transducer frequency, the minimum full-scale velocity is 75 $\mathrm{cm} / \mathrm{sec}$. The 128 point FFT used in the NeuroGuard analyzer provides 64-pixel resolution in both the antegrade and retrograde directions. Using the most sensitive scale, one pixel velocity resolution on the VGA monitor is thus $1.2 \mathrm{~cm} / \mathrm{sec}$ (i.e. $75 / 64$ ). Without filtering, a $4 \mathrm{~cm} / \mathrm{sec}$ flow would result in an easily distinguishable 4- to 5-pixel signal.

However, transcranial Doppler ultrasonography units use a high-pass filter to attenuate high-amplitude, lowfrequency acoustic artifact arising from tissue movement against the probe. Initially, we used diagnostic ultrasonographs with high-pass filters of $150 \mathrm{~Hz}(-3 \mathrm{~dB}$ roll-off frequency) or higher to measure low retrograde flows. In the worst case (i.e., complete sharp filter attenuation, not $-3 \mathrm{~dB}$ ), the minimum detectable velocity with such filters determined from the above equation is $5.8 \mathrm{~cm} / \mathrm{sec}$. This threshold exceeds that found in $25 \%$ of our cases. The more recently introduced $75 \mathrm{~Hz}$ high-pass filter now makes possible flow velocity measurements as low as 2.9 $\mathrm{cm} / \mathrm{sec}$ or $>2$ pixels, which is a small but discernable image. 